\title{
Behaviour of the plasma concentration of gelatinases and their tissue inhibitors in subjects with venous leg ulcers
}

\author{
G. Caimi*, F. Ferrara, M. Montana, I. Muratori, C. Amato, B. Canino, R. Lo Presti \\ and E. Hopps \\ Dipartimento Biomedico di Medicina Interna e Specialistica, Universitá di Palermo, Palermo, Italy
}

\begin{abstract}
Venous leg ulcers are common in subjects with chronic venous insufficiency. The increased intraluminal pressure causes alteration of the skin microcirculation, leukocyte activation and release of proteolytic enzymes leading to ulceration. An impaired expression and activity of matrix metalloproteases (MMPs) and their tissue inhibitors (TIMPs) might influence extracellular matrix degradation and deposition in chronic venous ulcers with the failure of the healing process. Our aim was to evaluate plasma concentration of gelatinases (MMP-2 and MMP-9) and their inhibitors (TIMP-1 and TIMP-2) in subjects with venous leg ulcers before and after the compression therapy. We enrolled 36 subjects (12 men and 24 women, mean age $67.38 \pm 12.7 \mathrm{yrs})$ with non-infected venous leg ulcers (CEAP C6), which underwent a color Duplex scan examination of the veins and arteries of the inferior limbs and were treated with a multi-layer bandaging system. The ulcer healing was obtained in 23 subjects only ( 9 men and 14 women). We evaluated, on fasting venous blood, the plasma levels of MMP-2, MMP-9, TIMP-1 and TIMP-2 using ELISA kit, before and after the treatment. We observed a significant increase in plasma concentration of gelatinases and their inhibitors and in MMP-2/TIMP-2 ratio in subjects with leg ulcers in comparison with normal controls. In subjects with healed ulcers we found a decrease in MMP-9 and TIMP-1 levels and in MMP-2/TIMP-2 ratio compared to the baseline values, although higher levels of all the examined parameters in comparison with normal controls. In conclusion, plasma MMPs profile is impaired in subjects with venous leg ulcers and it improves after the healing, persisting anyway altered in respect to healthy controls.
\end{abstract}

Keywords: Venous leg ulcers, MMP-2, MMP-9, TIMP-1, TIMP-2

\section{Introduction}

Venous leg ulcers associated with chronic venous insufficiency (CVI) affect approximately $1-1.5 \%$ of the general population and are responsible for a severe impairment of the quality of life and for elevated costs because of long treatment times [25].

The etiology of venous ulcers is still unclear, but it has been suggested that ulceration results from an increased intraluminal pressure that causes fibrin deposition around the capillaries with consequent leukocyte activation and release of proteolytic enzymes, responsible for tissue destruction. Venous leg ulcers in fact are characterized histologically by loss of the epidermidis, alterations of dermal cellular and matrix structures, inflammation, and by fibrin cuffs surrounding dermal capillary vessels and inhibiting the diffusion of oxygen and nutrients to the epidermidis $[4,16]$. Compression therapy is the gold standard for

\footnotetext{
${ }^{*}$ Corresponding author: G. Caimi, Dipartimento Biomedico di Medicina Interna e Specialistica, Universitá di Palermo, Palermo, Italy. E-mail: gregorio.caimi@unipa.it.
} 
the initial treatment of venous leg ulcers: by the application of graduated pressure to the leg, venous return improves reducing reflux [3]. As the pathogenesis of venous ulcer is associated with microcirculatory alterations and with an inflammatory response, compression stockings may improve healing rates and prevent ulcer recurrence $[4,32]$.

In the last decade many authors have described an altered pattern of matrix metalloproteases (MMPs) and their tissue inhibitors (TIMPs) in chronic venous insufficiency and in venous ulcers of the lower extremities [2, 4, 21, 23].

Matrix metalloproteinases are endopeptidases which activity consists principally of extracellular matrix protein degradation by cleavage of internal peptide bonds. They are secreted by all the cells present into the vascular wall, and especially by macrophages, and each MMP has a specific target substrate that defines its denomination, such as collagenases, including MMP-1, $-8,-13$, and -18 , gelatinases A and B (MMP-2 and -9), stromelysin-1 (MMP-3) and -2 (MMP-10), matrilysin-1 (MMP-7) and -2 (MMP-26) and metalloelastase (MMP-12) [1, 6, 17]. Most MMPs are secreted as precursors (zymogens) which are activated in the extracellular space by several proteases, oxidative stress metabolites, proinflammatory cytokines, and membrane-type MMPs. The activity of MMPs is regulated by the four tissue inhibitors of MMP (TIMPs): TIMP-1 inhibits MMP-1, MMP-3, MMP-7 and MMP-9, TIMP-2 inhibits especially MMP-2, TIMP-3 can inhibit both the gelatinases, while TIMP-4 inhibits MT-1 MMP and MMP-2 [1, $6,17]$. An imbalance between MMPs activation and inhibition may cause an uncontrolled proteolytic activity and an elevated turnover of the extracellular matrix resulting in leg ulcers formation and in impaired wound healing.

In severe chronic venous insufficiency Saito et al. [26] have observed elevated levels of MMP-1 and TIMP-1 and increased MMP-2 expression and activity in biopsies obtained from skin areas of venous stasis dermatitis, while other authors [33] have found higher concentrations of the active form of MMP-9 in the leg venous blood samples of subjects with CVI in comparison with controls.

Regarding the role of the gelatinases in chronic venous leg ulcers, Norgauer et al. [24] have shown an increased MMP-2 activity and a dermal overexpression of MMP-2 and membrane-tpe MMPs in biopsies from lesional tissue, while Moor et al. [22] have found significantly higher levels of active MMP-9 in the wound fluids and tissues of non-healers. Mwaura et al. [23] have examined MMP-2 and TIMP-2 in the wound fluid before and after 8 weeks of graduated compression bandaging treatment; they observed higher levels of MMP-2 and lower levels of TIMP-2 in non-healer subjects, although these data were not statistically significant. Beidler et al. [4] have examined a large range of MMPs (MMP-1, -2, -3, -7, -8, $-9,-12$ and -13 ) and TIMPs (TIMP-1 and -2) in leg ulcer tissue before and after 4 weeks of compression therapy and have described elevated MMPs (with the exception of MMP-7) and TIMP-1 levels and higher MMP-9 activity in chronic wounds; after therapy, they have observed a significant reduction of MMP$3,-8$, and -9 expression and a decrease in MMP-1, -2 , and -3 in more rapid healing ulcers. Also other authors have found higher levels of MMP-1 and MMP-8 in non-healing ulcers' biopsies and exudates [2], differently from Meyer et al. [21], who have observed higher levels of MMP-1 in biopsies from healing ulcers compared with resistant ulcers. This finding is unexpected considering that, in vitro, normal dermal fibroblasts incubated with chronic venous ulcer exudate have shown an increased expression of MMP-1 and MMP-3 and a reduced synthesis of TIMP-1 [31].

Up to now, only few studies have examined the plasma MMPs profile in subjects with chronic leg ulcers and also the systemic effects of the compression treatment on these markers of inflammation $[28,30]$.

In this research, we examined the plasma concentrations of the gelatinases and their tissue inhibitors in a group of patients with non-infected leg venous ulcers, and in a subgroup of these patients we examined 
the same parameter before and after the healing of venous ulcers obtained employing the compression therapy.

\section{Materials and methods}

We consecutively recruited 36 subjects ( 12 men and 24 women; mean age $67.38 \pm 12.7 \mathrm{yrs}$ ) with noninfected venous leg ulcers from those with venous diseases (CEAP: C6) referred to our Department. Before the inclusion in the study, all the patients underwent a color Duplex scan (CDS) examination performed using Philips HDI 5000 ultrasonography device with a 7-MHz probe. The ultrasound examination showed the status of the veins (superficial and deep) and of the arteries of the inferior limbs and the characteristics of the ulcers (post-thrombotic or varicose). Venous district evaluation was performed in supine and orthostatic position, while arterial district evaluation was performed in supine position. Using ultrasound examination we diagnosed the presence of superficial, deep valvular or perforating valvular incompetence and the partial or total obstruction of the veins. The valvular incompetence was diagnosed by the variations of the vein flow direction during the Valsalva and the compression and release maneuvers. We distinguished total from partial obstruction by a duplex definition. In total obstruction, the vein lumen is entirely occupied by thrombotic lesion and there is neither Doppler sound nor color-mapping imaging. On the contrary, in partial obstruction, we can discern the blood velocity and the color-mapping imaging in the vein lumen. We also measured the ankle-brachial index according to the current Transatlantic Intersociety Consensus guidelines to exclude the patients with arteriopathies of lower limbs, in which the application of an elastic bandage is not possible. Means and S.D. of laboratory parameters and duration of the disease are shown in Table 1.

The control group consisted of 30 subjects ( 14 women and 16 men, mean age $41.3 \pm 7.4$ years) selected from the hospital staff; control subjects were free of medical diseases as assessed by clinical history, physical examination, electrocardiography, and routine hematological and urine analysis.

All the subjects gave their informed consent before entering the study and the study was approved by the Ethical Committee.

On fasting venous blood we evaluated the plasma concentration of gelatinases (MMP-2 and MMP-9) and their inhibitors (TIMP-1 and TIMP-2) using respectively the Human MMP-2 ELISA and Human

Table 1

Means \pm S.D. and range of the main laboratory parameters and duration of the disease of the whole group of subjects with chronic venous

\begin{tabular}{lcc}
\hline & Means \pm S.D. & Range \\
\hline Glycaemia (mg/dl) & $86.19 \pm 6.34$ & $76-98$ \\
Creatininemia (mg/dl) & $0.94 \pm 0.09$ & $0.77-1.12$ \\
Total cholesterol (mg/dl) & $211.36 \pm 20.07$ & $123-248$ \\
HDL-cholesterol (mg/dl) & $55.69 \pm 11.30$ & $36-103$ \\
LDL-cholesterol (mg/dl) & $128.40 \pm 17.76$ & $51-157$ \\
Triglyceridemia (mg/dl) & $132.55 \pm 18.21$ & $82-158$ \\
WBC (cells/mm $\left.{ }^{3}\right)$ & $7915 \pm 351$ & $7200-9200$ \\
Albumin $(\mathrm{g} / \mathrm{dl})$ & $4.54 \pm 0.24$ & $3.98-4.9$ \\
Duration of the disease (months) & $7 \pm 1.78$ & $4-12$ \\
\hline
\end{tabular}


MMP-9 ELISA kit (Boster Biological Technology, LTD) and the Human TIMP-1 ELISA and Human TIMP-2 ELISA kit (Boster Biological Technology, LTD) in the entire group of subjects with venous leg ulcers.

All the patients with venous leg ulcers were treated with a multi-layer bandaging system consisting of two anelastic bandage impregnated with oxide paste and coumarin (PRONTOZINK CUMARINA cm $8 \times 5$ and $10 \times 5$ m PMA-Italia) and of two single stretch compression bandage (FISIODUR $\mathrm{cm} 8 \times 5$ and $10 \times 5 \mathrm{~m}$ PMA-Italia).

Before and after the compression treatment, we measured the same parameters in the subgroup of patients with healed ulcers (14 women and 9 men, mean age $66.08 \pm 12.33$ ).

\section{Statistical analysis}

Data were expressed as means \pm S.D.. The statistical difference between the whole group of subjects with venous leg ulcers and healthy controls was evaluated using the Student's $t$ test for unpaired data; the statistical difference regarding the plasma concentration of MMP-2, MMP-9, TIMP-1, and TIMP-2 before and after healing was studied with the Student's $t$ test for paired data. The statistical difference between the subgroup of subjects with healed leg venous ulcers and healthy controls was performed employing the Student's $t$ test for unpaired data. The null hypothesis was rejected for $p$ values $<0.05$.

\section{Results}

Examining the whole group of subjects with leg venous ulcers in comparison with healthy controls, we found a significant increase in the plasma concentrations of MMP-2, MMP-9, TIMP-1 and TIMP-2; we also found an increase in MMP-2/TIMP-2 ratio even if no variation regarding MMP-9/TIMP-1 ratio was observed between the two groups (Table 2).

In the subgroup of healed subjects we evaluated the plasma concentration of all these parameters before and after the healing of the venous ulcers and we observed that, after the healing, MMP-9, TIMP-2 and MMP-9/TIMP-1 ratio were decreased while no significant variation about MMP-2, TIMP-1 and MMP-2/TIMP-2 ratio was found (Table 3).

In addition, we also compared the subgroup of patients after the healing of leg ulcers with healthy controls and we found higher values of plasma concentrations of MMP-2, MMP-9, TIMP-1 and TIMP-2 and of MMP-2/TIMP-2 ratio in this subgroup than in healthy controls (Table 4).

Table 2

Means \pm S.D. of gelatinase and gelatinase inhibitor plasma concentrations in control subjects and in patients with venous ulcers

\begin{tabular}{lcc}
\hline & Control subjects & Patients with venous ulcers \\
\hline MMP-2 (ng/ml) & $28.66 \pm 4.15$ & $48.66 \pm 12.03^{* * *}$ \\
TIMP-2 (ng/ml) & $85.67 \pm 9.40$ & $118.5 \pm 9.15^{* * *}$ \\
MMP-9 (ng/ml) & $51.55 \pm 8.14$ & $109.1 \pm 18.73^{* * *}$ \\
TIMP-1 (ng/ml) & $32.05 \pm 4.80$ & $60.02 \pm 6.82^{* * *}$ \\
MMP-2/TIMP-2 & $0.338 \pm 0.059$ & $0.411 \pm 0.099^{* * *}$ \\
MMP-9/TIMP-1 & $1.652 \pm 0.376$ & $1.850 \pm 0.421$
\end{tabular}

${ }^{* * * *} p<0.001$ vs control subjects (Student's “ $t$ " test for unpaired data). 
Table 3

Means \pm S.D. of gelatinase and gelatinase inhibitor plasma concentrations in patients whose ulcers heal, at baseline and after healing

\begin{tabular}{llc}
\hline & \multicolumn{1}{c}{ Baseline } & After ulcer healing \\
\hline MMP-2 (ng/ml) & $48.07 \pm 12.40$ & $44.04 \pm 10.70$ \\
TIMP-2 (ng/ml) & $117.4 \pm 8.23$ & $112.3 \pm 7.49^{*}$ \\
MMP-9 (ng/ml) & $109.4 \pm 19.2$ & $93.1 \pm 20.8^{* * *}$ \\
TIMP-1 (ng/ml) & $59.09 \pm 6.78$ & $57.66 \pm 4.74$ \\
MMP-2/TIMP-2 & $0.410 \pm 0.105$ & $0.394 \pm 0.100$ \\
MMP-9/TIMP-1 & $1.888 \pm 0.456$ & $1.628 \pm 0.404^{* *}$ \\
\hline
\end{tabular}

${ }^{*} p<0.05 ;{ }^{* *} p<0.01 ;{ }^{* * *} p<0.001$ vs baseline (Student's " $t$ " test for paired data).

Table 4

Means \pm S.D. of gelatinase and gelatinase inhibitor plasma concentrations in control subjects and in patients with healed venous ulcers

\begin{tabular}{lcc}
\hline & Control subjects & Patients with healed venous ulcers \\
\hline MMP-2 (ng/ml) & $28.66 \pm 4.15$ & $44.04 \pm 10.70^{* * *}$ \\
TIMP-2 (ng/ml) & $85.67 \pm 9.40$ & $112.3 \pm 7.49^{* * *}$ \\
MMP-9 (ng/ml) & $51.55 \pm 8.14$ & $93.1 \pm 20.8^{* * *}$ \\
TIMP-1 (ng/ml) & $32.05 \pm 4.80$ & $57.66 \pm 4.74^{* * *}$ \\
MMP-2/TIMP-2 & $0.338 \pm 0.059$ & $0.394 \pm 0.100^{*}$ \\
MMP-9/TIMP-1 & $1.652 \pm 0.376$ & $1.628 \pm 0.404$ \\
\hline
\end{tabular}

${ }^{*} p<0.05 ;{ }^{* * *} p<0.001$ vs control subjects (Student's “ $t$ " test for unpaired data).

\section{Discussion}

In previous papers regarding several venous diseases, such as deep venous thrombosis [9, 19], post-thrombotic syndrome [7, 8] and leg venous ulcers [20], we demonstrated, especially after in vitro activation with 4-phorbol 12-myristate 13-acetate (PMA) and with N-formyl-methionyl-leucyl-phenilalanine (fMLP), an evident alteration of the two parameters that reflect the rheology of the polymorphonuclear cells: the membrane fluidity, examined employing the fluorescent probe TMA-DPH, and the cytosolic calcium concentration, explored using the fluorescent probe Fura-2AM. In subjects with deep venous thrombosis [9] and leg venous ulcers [20] we also found an altered profile of the polymorphonuclear integrins (CD11a, CD11b, CD11c and CD18) at baseline and after activation with PMA and fMLP.

These previous reports underline clearly the role played by polymorphonuclear cells in the pathogenesis and in the progression of chronic venous diseases and especially in venous ulcers.

The results of this study add other information regarding the complex role of inflammation in venous ulcers. Up to now only few papers have regarded the evaluation of the plasma concentrations of gelatinases and their tissue inhibitors in this clinical condition $[2,28,30]$ whereas more data are available regarding MMPs and their inhibitors in biopsies from damaged tissue [24] or in wound fluids of non-healers [22] and their behavior before and after some weeks of compression therapy [5, 23]. 
Our results seem to indicate that, although at baseline the gelatinases and their inhibitors are significantly increased in patients with venous leg ulcers, the healing of these ulcers, obtained with compression therapy, is associated with reduced plasma levels of MMP-9, TIMP-2 and also of MMP-9/TIMP-1 ratio. However, also in this subgroup of healed patients, the concentrations of gelatinases and their inhibitors are statistically elevated in comparison with healthy controls.

Our data, together with literature data $[2,28,30]$, besides to confirm the increase in plasma levels of MMPs and their inhibitors in venous ulcers, suggest that the healing partially influences their behavior. It is not possible to exclude a bidirectional relationship between the progression of chronic venous insufficiency towards ulcers and the trend of some MMPs, especially of those explored in the lesion tissue where they seem to play, directly or indirectly (increased transforming growth factor â1, dermal fibroblast production), a specific role in the dynamics of extracellular matrix turnover, that may be retained responsible respectively for venous ulcers genesis and repair.

At this regard it is also useful to underline the behavior of the plasma levels of tissue inhibitors in subjects with venous ulcers. In fact, from our data it is incontestable that the increase in gelatinases is accompanied by an analogous increase of their tissue inhibitors. This apparent dysregulation between gelatinases and their inhibitors might be imputable to their co-secretion or to a compensatory effect [13].

Another aspect that must be underlined is that in this research we examined only the plasma levels of MMP-2 and MMP-9 and not their activity and this distinction is necessary especially considering the possible role of MMPs in this clinical condition.

Bearing in mind this hypothesis, an obvious consideration may regard the possibility to pharmacologically interfere on these parameters in leg venous ulcers. In literature, up to now, some papers have regarded the action of the tetracyclines, and in particular of doxycycline, on MMPs in animal models of transient global cerebral ischemia [18] and vascular alterations related to arterial hypertension [10]. The same antibiotics seem to modulate MMPs activity, irrespective of their antimicrobial activity, in subjects with periodontitis, that is considered a risk factor for atherosclerotic cardiovascular events [14], as well as in cardiovascular diseases [11]. Doxacycline has been used at low doses also in the treatment of chronic venous ulcers [29] and it seems to accelerate their healing, even if it is not possible to exclude a role of the antimicrobial activity in the venous ulcers repair.

In the treatment of venous ulcers other molecules able to interfere with MMPs activity might be employed: dipyridamole [15], an antiplatelet agents, cilostazol [12], a specific phosphodiesterase type III inhibitor with anti-atherosclerotic effect, and polyphenols [27], able to regulate the MMPs, and in particular the MMP-9.

In conclusion, the plasma MMPs profile is impaired in subjects with venous leg ulcers and it improves after their healing, persisting anyway altered in comparison with healthy controls. Besides the compression therapy, the employing of some molecules able to interfere with MMPs expression might be useful in venous ulcers treatment.

This research complies with the requirement for ethical publication in Clinical Hemorheology and Microcirculation as published in Clin Hemorheol Microcirc. 2010;44(1):1-2.

\section{References}

[1] C. Amãlinei, I.D. Cãruntu and R.A. Bãlan, Biology of metalloproteinases, Rom J Morphol Embryol 48 (2007), 323-334.

[2] B. Amato, G. Coretti, R. Compagna, M. Amato, G. Buffone, D. Gigliotti, R. Grande, R. Serra and S. de Franciscis, Role of matrix metalloproteinases in non-healing venous ulcers, Int Wound $J$ (2013), in press. 
[3] R.L. Ashby, R. Gabe, S. Ali, U. Adderley, J.M. Bland, N.A. Cullum, J.C. Dumville, C.P. Iglesias, A.R. Kang'ombe, M.O. Soares, N.C. Stubbs and D.J. Torgerson, Clinical and cost-effectiveness of compression hosiery versus compression bandages in treatment of venous leg ulcers (Venous leg Ulcer Study IV, VenUS IV): A randomised controlled trial, Lancet (2013), in press.

[4] S.K. Beidler, C.D. Douillet, D.F. Berndt, B.A. Keagy, P.B. Rich and W.A. Marston, Inflammatory cytokine levels in chronic venous insufficiency ulcer tissue before and after compression therapy, J Vasc Surg 49 (2009), 1013-1020.

[5] S.K. Beidler, C.D. Douillet, D.F. Berndt, B.A. Keagy, P.B. Rich and W.A. Marston, Multiplexed analysis of matrix metalloproteinases in leg ulcer tissue of patients with chronic venous insufficiency before and after compression therapy, Wound Repair Regen 16 (2008), 642-648.

[6] D. Bourboulia and W.G. Stetler-Stevenson, Matrix metalloproteinases (MMPs) and tissue inhibitors of metalloproteinases (TIMPs): Positive and negative regulators in tumor cell adhesion, Semin Cancer Biol 20 (2010), 161-168.

[7] G. Caimi, B. Canino, F. Ferrara, M. Montana and R. Lo Presti, Leucocyte rheology at baseline and after activation in post-phlebitic syndrome, Phlebology 14 (1999), 100-104.

[8] G. Caimi, B. Canino, F. Ferrara, M. Montana, F. Raimondi and R. Lo Presti, Leukocyte rheology before and after chemotactic activation in some venous diseases, Eur J Endovasc Surg 18 (1999), 411-416.

[9] G. Caimi, M.G. Tozzi Ciancarelli, F. Ferrara, M. Montana, V. Calandrino, B. Canino and R. Lo Presti, Deep venous thrombosis: Behaviour of the polymorphonuclear leukocyte integrin pattern at baseline and after in vitro activation, Clinical Hemorheol Microcirc 33 (2005), 11-17.

[10] M.M. Castro, A.D. Kandasamy, N. Youssef and R. Schulz, Matrix metalloproteinase inhibitor properties of tetracyclines: Therapeutic potential in cardiovascular diseases, Pharmacol Res 64 (2011), 551-560.

[11] M.M. Castro, J.E. Tanus-Santos and R.F. Gerlach, Matrix metalloproteinases: Targets for doxycycline to prevent the vascular alterations of hypertension, Pharmacol Res 64 (2011), 567-572.

[12] S.Y. Chuang, S.H. Yang, T.Y. Chen and J.H. Pang, Cilostazol inhibits matrix invasion and modulates the gene expressions of MMP-9 and TIMP-1 in PMA-differentiated THP-1 cells, Eur J Pharmacol 670 (2011), 419-426.

[13] R. Deardorff and F.G. Spinale, Cytokines and matrix metalloproteinases as potential biomarkers in chronic heart failure, Biomark Med 3 (2009), 513-523.

[14] Y. Gu, H.M. Lee, T. Sorsa, A. Salminen, M.E. Ryan, M.J. Slepian and L.M. Golub, Non-antibacterial tetracyclines modulate mediators of periodontitis and atherosclerotic cardiovascular disease: A mechanistic link between local and systemic inflammation, Pharmacol Res 64 (2011), 573-579.

[15] S. Guo, M. Stins, M. Ning and E.H. Lo, Amelioration of inflammation and cytotoxicity by dipyridamole in brain endothelial cells, Cerebrovasc Dis 30 (2010), 290-296.

[16] Y. Herouy, D. Trefzer, U. Zimpfer, E. Schöpf, W. Wanscheidt and J. Norgauer, Matrix metalloproteinases and venous leg ulceration, Eur J Dermatol 10 (2000), 173-180.

[17] A. Jaiswal, A. Chhabra, U. Malhotra, S. Kohli and V. Rani, Comparative analysis of human matrix metalloproteinases: Emerging therapeutic targets in diseases, Bioinformation 6 (2011), 23-30.

[18] H. Lee, J.W. Park, S.P. Kim, E.H. Lo and S.R. Lee, Doxycycline inhibits matrix metalloproteinase-9 and laminin degradation after transient global cerebral ischemia, Neurobiol Dis 34 (2009), 189-198.

[19] R. Lo Presti, F. Ferrara, B. Canino, M. Montana and G. Caimi, Deep venous thrombosis: Leukocyte rheology at baseline and after in vitro activation, Haemostasis 30 (2000), 168-173.

[20] R. Lo Presti, F. Ferrara, B. Canino, A. Valenti, F. Raimondi and G. Caimi, Fluiditá di membrana, calcio citosolico ed assetto integrinico dei leucociti polimorfonucleati in pazienti con ulcere venose, Minerva Cardioangiologica S1 (2006), 246-248.

[21] F.J. Meyer, K.G. Burnand, S. Abisi, J.M. Tekoppele, B. van Els and A. Smith, Effect of collagen turnover and matrix metalloproteinase activity on healing of venous leg ulcers, Br J Surg 95 (2008), 319-325.

[22] A.N. Moor, D.J. Vachon and L.J. Gould, Proteolytic activity in wound fluids and tissues derived from chronic venous leg ulcers, Wound Repair Regen 17 (2009), 832-839.

[23] B. Mwaura, B. Mahendran, N. Hynes, D. Defreitas, G. Avalos, T. Adegbola, M. Adham, C.E. Connolly and S. Sultan, The impact of differential expression of extracellular matrix metalloproteinase inducer, matrix metalloproteinase-2, tissue inhibitor of matrix metalloproteinase-2 and PDGF-AA on the chronicity of venous leg ulcers, Eur J Vasc Endovasc Surg 31 (2006), 306-310.

[24] J. Norgauer, T. Hildenbrand, M. Idzko, E. Panther, E. Bandemir, M. Hartmann, W. Vanscheidt and Y. Herouy, Elevated expression of extracellular matrix metalloproteinase inducer (CD147) and membrane-type matrix metalloproteinases in venous leg ulcers, Br J Dermatol 147 (2002), 1180-1186. 
[25] T.F. O’Donnell Jr and E.M. Balk, The need for an Intersociety Consensus Guideline for venous ulcer, J Vasc Surg 54 (2011), 83S-90S.

[26] S. Saito, M.J. Trovato, R. You, B.K. Lal, F. Fasehun, F.T. Padberg Jr, R.W. Hobson 2nd, W.N. Durán and P.J. Pappas, Role of matrix metalloproteinases 1,2, and 9 and tissue inhibitor of matrix metalloproteinase-1 in chronic venous insufficiency, J Vasc Surg 34 (2001), 930-938.

[27] E. Scoditti, N. Calabriso, M. Massaro, M. Pellegrino, C. Storelli, G. Martines, R. De Caterina and M.A. Carluccio, Mediterranean diet polyphenols reduce inflammatory angiogenesis through MMP-9 and COX-2 inhibition in human vascular endothelial cells: A potentially protective mechanism in atherosclerotic vascular disease and cancer, Arch Biochem Biophys 527 (2012), 81-89.

[28] R. Serra, G. Buffone, D. Falcone, V. Molinari, M. Scaramuzzino, L. Gallelli and S. de Franciscis, Chronic venous leg ulcers are associated with high levels of metalloproteinases-9 and neutrophil gelatinase-associated lipocalin, Wound Repair Regen 21 (2013), 395-401.

[29] R. Serra, L. Gallelli, G. Buffone, V. Molinari, D.M. Stillitano, C. Palmieri and S. de Franciscis, Doxycycline speeds up healing of chronic venous ulcers, Int Wound $J$ (2013), in press.

[30] R. Serra, R. Grande, G. Buffone, V. Molinari, P. Perri, A. Perri, B. Amato, M. Colosimo and S. de Franciscis, Extracellular matrix assessment of infected chronic venous leg ulcers: Role of metalloproteinases and inflammatory cytokines, Int Wound $J$ (2014), in press.

[31] K. Subramaniam, C.M. Pech, M.C. Stacey and H.J. Wallace, Induction of MMP-1, MMP-3 and TIMP-1 in normal dermal fibroblasts by chronic venous leg ulcer wound fluid, Int Wound J 5 (2008), 79-86.

[32] J.C. Tang, W.A. Marston and R.S. Kirsner, Wound Healing Society (WHS) venous ulcer treatment guidelines: What's new in five years? Wound Repair Regen 20 (2012), 619-637.

[33] P. Zamboni, G. Scapoli, V. Lanzara, M. Izzo, P. Fortini, R. Legnaro, A. Palazzo, S. Tognazzo and D. Gemmati, Serum iron and matrix metalloproteinase-9 variations in limbs affected by chronic venous disease and venous leg ulcers, Dermatol Surg 31 (2005), 644-649. 